\author{
Alexander M. McKinney \\ Sean O. Casey \\ Mehmet Teksam \\ Leandro T. Lucato \\ Maurice Smith \\ Charles L. Truwit \\ Stephen Kieffer
}

\section{Carotid bifurcation calcium and correlation with percent stenosis of the internal carotid artery on CT angiography}

Received: 30 December 2003

Accepted: 4 September 2004

Published online: 14 January 2005

(c) Springer-Verlag 2005
Presented at the 41st Annual Meeting of the American Society of Neuroradiology, Washington D.C., 2003. Sean Casey, MD and Charles Truwit, MD are members of the Medical Advisory Board of Vital Images (Plymouth, Minnesota), the company that developed the Vitrea 2 software.

A. M. McKinney $(\bowtie) \cdot S$. O. Casey M. Teksam - C. L. Truwit - S. Kieffer Departments of Radiology, University of Minnesota Medical School, and Hennepin County Medical Center, Minneapolis, MN 55455, USA

E-mail: mckin022@umn.edu

Tel.: + 1-612-2734092

Fax: + 1-612-6248495

L. T. Lucato

Clinics Hospital of the University of Sao Paulo, Sao Paulo, Brazil

M. Smith

Department of Biomedical Engineering, Johns Hopkins University, Baltimore, MD 21218, USA
Abstract The aim of this paper was to determine the correlation between calcium burden (expressed as a volume) and extent of stenosis of the origin of the internal carotid artery (ICA) by CT angiography (CTA). Previous studies have shown that calcification in the coronary arteries correlates with significant vessel stenosis, and severe calcification (measured by CT) in the carotid siphon correlates with significant (greater than $50 \%$ stenosis) as determined angiographically. Sixty-one patients (age range 50-85 years) underwent $\mathrm{CT}$ of the neck with intravenous administration of iodinated contrast for a variety of conditions. Images were obtained with a helical multidetector array CT scanner and reviewed on a three-dimensional workstation. A single observer manipulated window and level to segment calcified plaque from vascular enhancement in order to quantify vascular calcium volume (cc) in the region of the bifurcation of the common carotid artery/ICA origin, and to measure the extent of ICA stenosis near the origin. A total of 117 common carotid artery bifurcations were reviewed. A

"significant" stenosis was defined arbitrarily as $>40 \%$ (to detect lesions before they become hemodynamically significant) of luminal diameter on CTA using NASCETlike criteria. All "significant" stenoses (21 out of 117 carotid bifurcations) had measurable calcium. We found a relatively strong correlation between percent stenosis and the calcium volume (Pearson's $r$ $=0.65, P<0.0001)$. We also found that there was an even stronger correlation between the square root of the calcium volume and the percent stenosis as measured by CTA $(r=$ $0.77, P<0.0001)$. Calcium volumes of $0.01,0.03,0.06,0.09$ and $0.12 \mathrm{cc}$ were used as thresholds to evaluate for a "significant" stenosis. A receiver operating characteristic (ROC) curve demonstrated that thresholds of $0.06 \mathrm{cc}$ (sensitivity $88 \%$, specificity $87 \%$ ) and $0.03 \mathrm{cc}$ (sensitivity $94 \%$, specificity $76 \%$ ) generated the best combinations of sensitivity and specificity. Hence, this preliminary study demonstrates a relatively strong relationship between volume of calcium at the carotid bifurcation in the neck (measured by CT) and percent stenosis of the ICA below the skull base (as measured by CTA). Use of calcium volume measurements as a threshold may be both sensitive and specific for the detection of significant ICA stenosis. The significance of the correlation between calcium volume and ICA stenosis is that potentially a "score" can be obtained that will identify those at risk for high grade carotid stenosis.

Keywords Calcium · Carotid arteries - Calcification - Computed tomography $(\mathrm{CT}) \cdot \mathrm{CT}$ angiography $(\mathrm{CTA}) \cdot$ Carotid stenosis 


\section{Introduction}

Atherosclerotic disease in the coronary and carotid arteries is a significant cause of morbidity and mortality [1]. Coronary and carotid artery atherosclerotic disease share similar risk factors and are often coexistent in the at-risk population, especially diabetics, hypertensive patients, smokers, patients with hypercholesterolemia and those of advanced age [2-4]. Calcification in the coronary arteries has been correlated with significant vessel stenosis (defined as greater than 50\%) [5]. Severe calcification in the carotid siphon has also been associated with a greater than $50 \%$ carotid stenosis [6]. Coronary calcium scoring has been implemented for early detection of stenoses in high risk or symptomatic patients, and usually focuses on thresholds of density measurements obtained by helical CT or electron beam CT (EBCT) [5, 7, 8], although this technique has encountered controversy due to being sensitive $(88 \%)$ but not quite as specific (52\%) [5].

Ultrasound and MRA are commonly used non-invasive methods to evaluate for carotid stenosis in an at-risk population, and both modalities have been demonstrated to be sensitive for the detection of carotid stenosis, although ultrasound has been described as slightly less accurate and specific than MRA, particularly in patients with less than $70 \%$ stenosis $[9,10]$. CTA has a proven strong correlation with percent stenosis in evaluating for internal carotid artery (ICA) stenosis near the carotid bifurcation, relative to the gold-standard conventional catheter angiography [11-13]. However, CTA is not typically used as the initial evaluation for carotid stenosis due to expense and risks associated with intravenous administration of iodinated contrast material.

The primary goal of this study was to test the hypothesis that a sample volume of calcium at the carotid bifurcation may correlate with percent luminal stenosis. CTA was used as the relative gold standard in our study to evaluate the percent of luminal internal carotid stenosis near the bifurcation. As a preliminary study to determine the feasibility of using carotid calcium volume to detect "significant" stenoses, we studied a series of routine post-intravenous contrast neck CT examinations performed on a multidetector CT scanner. The data sets obtained from these scans allowed for simultaneous calcium volume measurements while also including the CT angiographic data for stenosis measurements.

\section{Materials and methods}

Sixty-one consecutive patients (age range 50-85 years, mean 63 years) were included in the study, all of whom underwent clinically indicated neck CT with a helical multidetector array (MDA) CT scanner following a bolus of intravenous administration of iodinated con- trast. Age (equal to or greater than 50 years) was the only significant factor for inclusion in this study. Patients were not subselected for diabetes or hypertension or other risk factors known to accelerate atherosclerotic disease. These examinations were performed for various indications, mostly for the purpose of evaluating head and neck cancer. None of the examinations was performed for suspected carotid occlusive disease. Thirtyeight females and 23 males were included. One patient with a known large invasive neck malignancy was excluded on the side of carotid encasement (right side), since it was difficult to distinguish carotid lumen throughout most of the neck. In four patients with known carotid endarterectomy, the side of involvement was also excluded on the side of surgery (two on the right, two on the left), since we considered the extent of disease to be unrepresentative of the true or past calcium burden; however, we included the non-operative side in all four cases. Hence, a total of five carotid bifurcations were excluded (out of 122 possible carotids in $61 \mathrm{pa}-$ tients).

All patients were evaluated with post-contrast neck CT studies, which were obtained on a quad detector array system (Siemens Volume Zoom; Forcheim, Germany). Scanning was performed using $120 \mathrm{kVp}$ with a maximum tube current of $230 \mathrm{~mA}$ (adjustable effective $\mathrm{mA}$ depending on patient body habitus). Scanning time was approximately $15 \mathrm{~s}$, and images were obtained during a 15-20 s breath hold. Scans were performed in a caudal-cranial direction from the level of the aortic arch up to $1 \mathrm{~cm}$ above the level of the sella in order to include the Circle of Willis. Source images were displayed with a 20-24 cm field of view depending on patient body habitus. A 22 gauge catheter or larger was used for injection of contrast medium in peripheral veins in either the antecubital region, forearm, or hand, for a total of $150 \mathrm{cc}$. Pre-intravenous contrast scans were not obtained. Non-ionic contrast material (ioversol $320 \mathrm{mg} / \mathrm{ml}$; Mallinckrodt, St Louis, Miss., USA) was administered in the following fashion: $50 \mathrm{cc}$ were injected manually prior to obtaining a scout topogram, and then scan range was set. Within minutes after the manual injection, the power injector was connected to the catheter, and contrast infusion started at a rate of $3 \mathrm{cc} / \mathrm{s}$ for the first $70 \mathrm{cc}$ and $2 \mathrm{cc} / \mathrm{s}$ for the last $30 \mathrm{cc}$. Delay between injection and scan was $30 \mathrm{~s}$. If significant dental amalgam was noted on the scout topogram, power injection rate was $3 \mathrm{cc} / \mathrm{s}$ for the first $40 \mathrm{cc}, 1.5 \mathrm{cc} / \mathrm{s}$ for the next $35 \mathrm{cc}$, and after a $20 \mathrm{~s}$ delay (and reangling of scan plane through the region of the mouth), the last $25 \mathrm{cc}$ was power injected at a rate of $2.8 \mathrm{cc} / \mathrm{s}, 1 \mathrm{~mm}$ collimation was used to produce $4 \mathrm{~mm}$ thick images for routine clinical use. Raw data were then retrospectively reconstructed to $1 \mathrm{~mm}$ thick sections at $0.5 \mathrm{~mm}$ intervals for use in calcium volume measurement and CTA evaluation of the carotid arteries. 
Fig. 1 a and b Maximum intensity display CTA coronal view (a) and sagittal view of the left side (b) demonstrates the carotid bifurcation calcium bilaterally (yellow arrows). There is also a small amount of calcified plaque more cephalad in the right internal carotid artery (red arrows). Decent quality $\mathrm{CT}$ angiographic images such as these were obtainable from the multidetector neck CT raw data. Note that the carotid calcifications are easily distinguishable from the arterial lumen due to the higher Hounsfield unit range of calcium. c (right and left carotids) Prior to carotid calcium measurement, the 3D data set was sculpted to a subvolume to exclude bony calcium related to spine, thyroid, and hyoid cartilage. d A density threshold was then visually adjusted (within a range of window 10-20 HU and level 250-500 HU) to include only calcium within the volume (thus excluding luminal contrast enhancement). Volume measurements were then calculated with an automated feature of the software. This patient has a left carotid calcium volume of $0.13 \mathrm{cc}$, and a right calcium volume of $0.10 \mathrm{cc}$. Note the small amount of calcium more cepahalad in the right ICA (red arrow). e Measurements of stenotic right and left ICA luminal diameter. CTA stenosis measurements were obtained in a NASCET-like fashion by measurement of ICA luminal diameter at the point of maximum stenosis (same patient as a-d). Non-calcified plaque (yellow arrow) was distinguishable from calcified plaque in this patient. f Measurements of the normal right and left ICA luminal diameter. At a level $2 \mathrm{~cm}$ or greater cephalad to the maximal stenosis, the more normal ICA diameter is obtained. This patient had an approximately $45 \%$ stenosis on the right, and $43 \%$ on the left
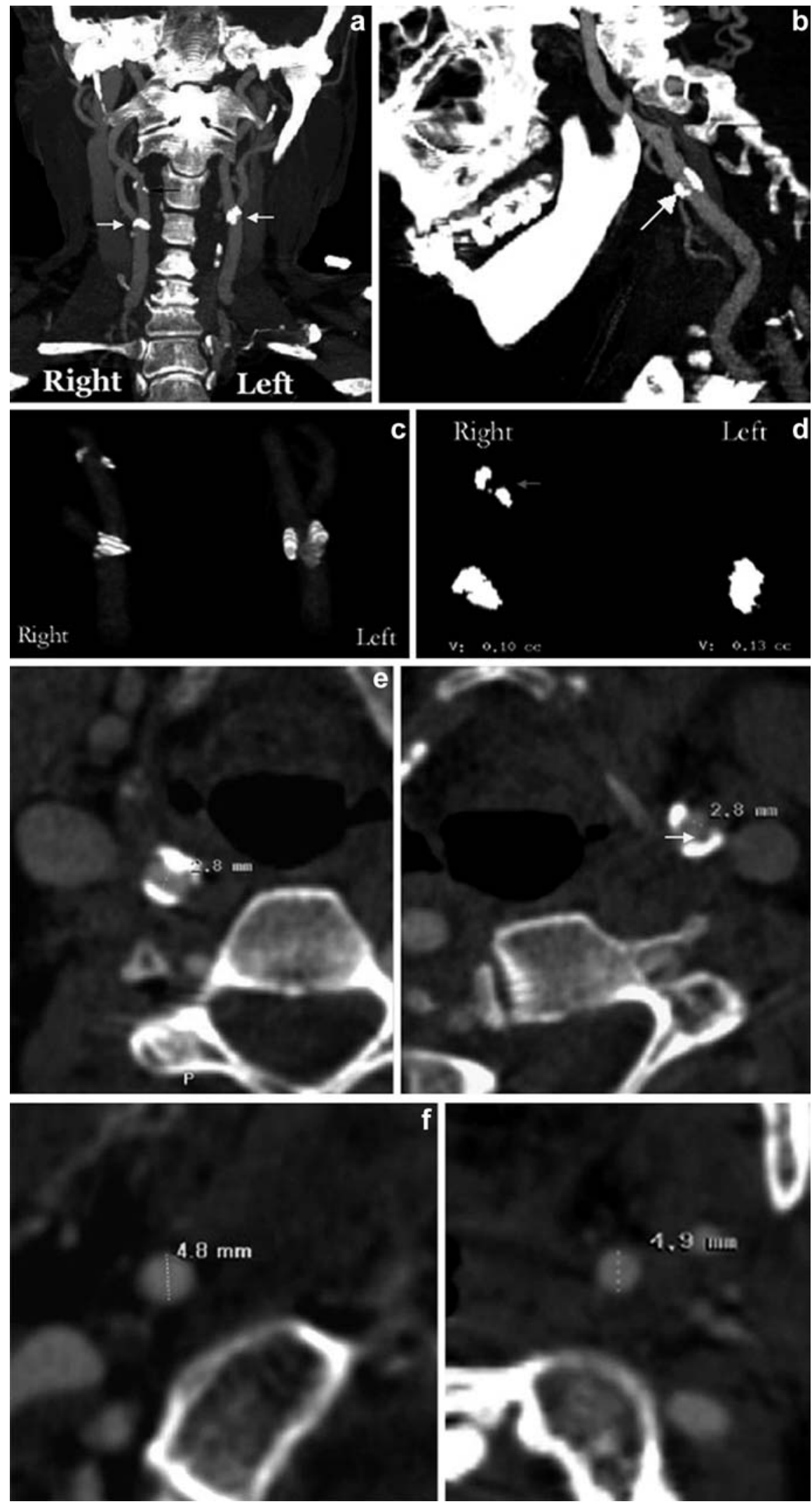
The reconstructed raw data were then sent to a Vitrea 2 workstation (Vital Images, Plymouth, Minn., USA) for evaluation of CTA and quantification of total vascular calcium volume by a single observer. The observer was able to view vessels in an infinite number of multiplanar views, including oblique angulations. The observer would first evaluate using various windows and levels for mass, trauma or other extravascular etiology that could alter visualization or measurement of the carotid bifurcation (Fig. 1a, b).

For the purpose of carotid bifurcation calcium burden measurement, a volume was manually "sculpted" from 2 above to $2 \mathrm{~cm}$ below the carotid bifurcation while taking care not to include thyroid cartilage, cricoid cartilage, or styloid process calcifications (Fig. 1c). This "sculpting" consisted of drawing the area intended for inclusion on serial axial images, so that other sources of calcification/bone (e.g. thyroid/cricoid cartilage and vertebra) were outside of the drawn area and hence excluded from the resulting sculpted volume. External carotid calcification near the bifurcation was not separated from that in the internal carotid artery while sculpting. After sculpting, the window and level were then manually altered to maximize the amount of visualized calcium (level 250-500 HU, window 10-20 HU) while not including vascular contrast (Fig. 1d). This combination of sculpting and windowing would result in visualization of only the bright/dense bifurcation calcium. Using a volume calculation function available on the workstation, plaque burden was calculated in cc. The software would automatically calculate the remaining volume (in cc) of the visualized calcium by the press of a button. The smallest plaque volume that can be calculated by the software is $0.01 \mathrm{cc}$.

After excluding extraneous cause of carotid involvement (such as tumor encasement/invasion), bifurcation vascular anatomy was evaluated by manipulating window/level (window 900-1200 HU, level 300-500 HU) to view calcified and non-calcified plaque and measure internal carotid stenoses. Calcified plaque was easily distinguished from non-calcified plaque by manually and visually manipulating window/level. The single reader was not blinded to results of calcium volume determination before measuring degree of stenosis. All measurements of stenosis were obtained on $1 \mathrm{~mm}$ thickness images to minimize volume averaging. To avoid overestimation of luminal diameter, the images used to measure luminal diameter stenosis were always obtained from an image plane perpendicular $\left(90^{\circ}\right)$ to the axis of the vessel. This often required reorientation of the image plane. Vascular luminal contrast enhancement was easily separated visually from calcified plaque (if present) by manual manipulation of window/level settings. This manipulation was monitored closely to avoid accidental inclusion of vascular enhancement in the calcium volume. To evaluate for the presence of stenosis, the observer measured the cross-sectional luminal diameter in millimeters at the narrowest location (Fig. 1e) (even in the absence of significant plaque) and measured the more normal/non-stenotic internal carotid artery diameter at least $2 \mathrm{~cm}$ above the site of narrowing/plaque (Fig. 1f). Thus, an attempt was made to adhere to North American Symptomatic Carotid Endarterectomy Trial (NASCET) criteria as closely as possible [14] when performing stenosis measurements. Locations of narrowing in the ICA were evaluated at any level from the carotid bifurcation to the skull base; common carotid artery narrowing and intracranial ICA narrowing were not evaluated in this study. Images of the final calcium burden volume and measurements of luminal diameter were stored for future reference. A single observer initially evaluated all studies, although a second observer re-evaluated those patients with "significant" stenoses.

For statistical analysis, the sensitivity and specificity as well as a receiver operating characteristic (ROC) curve of the study data were obtained at five arbitrary volume thresholds of calcium burden: $0.01,0.03,0.06$, 0.09 , and $0.12 \mathrm{cc}$. We arbitrarily used $40 \%$ as our definition for "significant stenosis" in order to detect a stenotic lesion before the stenosis reached 50\% (which is commonly considered the threshold at which a stenosis is hemodynamically significant). We considered a positive test one in which the patient had a greater than $40 \%$ luminal stenosis in one or both of the ICAs in the presence of the calcium burden threshold at which we were testing. A Pearson's correlation coefficient $(r)$ was calculated to assess for degree of correlation between calcium volume and percent stenosis. The correlation coefficient $(r)$ can range from -1.0 to 1.0 , where -1.0 is a perfect negative (inverse) correlation, 0.0 is no correlation, and 1.0 is a perfect positive correlation. Typically, an $r$ of $0.4-0.7$ is considered a moderate correlation and $0.7-0.9$ is a strong correlation. The statistical significance of $r$ was tested using a $t$-test. A statistician reviewed the data and these calculations.

\section{Results}

Vascular calcification was not difficult to distinguish and segment from luminal carotid contrast enhancement on the workstation. Total time to measure calcium burden and stenosis was approximately $2-5 \mathrm{~min}$ per side, depending largely on the tortuosity of the vessel and the degree of calcification. Thirty-five of the 61 patients in this study demonstrated measurable carotid bifurcation calcium burden greater than $0 \mathrm{cc}$ (27 on the right, 30 on the left), 21 of whom had bilateral calcification. We noted that the computer truncated two cases of very tiny amounts of visualized 
calcium (apparent to the observer) to $0 \mathrm{cc}$. Carotid bifurcation calcium ranged from 0 to $0.76 \mathrm{cc}$. The mean calcium burden of all carotid bifurcations was $0.053 \mathrm{cc}$ (SD 0.112).

CTA measures of ICA stenoses ranged from 0 to 99\% (mean 14.3\%). All measurable stenoses (38 carotids in 25 patients with a stenosis $>0 \%$ ) except one had detectable calcium, and this exception had a stenosis of only $19 \%$. Nevertheless, this patient had measurable calcium on the opposite side $(0.13 \mathrm{cc}$ with $38 \%$ stenosis on the opposite side). The greatest carotid bifurcation calcium volume without measurable stenosis by NASCET criteria was $0.17 \mathrm{cc}$. A "significant" stenosis (which we defined as $>40 \%$ ) was seen in 16 patients (for a total of 21 carotids), where five patients had bilateral "significant" stenoses. For the "significant" stenoses, the mean calcium burden was $0.215 \mathrm{cc}$ (SD 0.204). All "significant" stenoses demonstrated a measurable calcium burden, although one patient with prior carotid endarterectomy had a $99 \%$ stenosis and only mild calcium burden $(0.04 \mathrm{cc})$. This was one of two cases of $99 \%$ stenosis in this study, both of which had previously undergone prior carotid endarterectomy on the opposite side. Three carotids with greater than $70 \%$ stenosis were detected in three different patients.

Using calcium volumes of $0.01,0.03,0.06,0.09$ and $0.12 \mathrm{cc}$ as thresholds for evaluation for "significant" stenoses, the sensitivity, specificity, positive predictive value, and negative predictive value were calculated (Table 1). A ROC curve was also generated using the sensitivity and specificity (Fig. 2). The sensitivity was $100 \%$ for both sides when using a minimum threshold of $0.01 \mathrm{cc}$, but the specificity was only $56 \%$. The specificity was $93 \%$ for both sides when using $0.12 \mathrm{cc}$ as a threshold, but the sensitivity was only $56 \%$. Thresholds of $0.06 \mathrm{cc}$ (sensitivity $88 \%$, specificity $87 \%$ ) and $0.03 \mathrm{cc}$ (sensitivity $94 \%$, specificity $76 \%$ ) generated the best combination of sensitivity and specificity.

Table 1 Sensitivities and specificities at the five calcium volume thresholds that were used to evaluate for a "significant" stenosis. $P P V$ positive predictive value, $N P V$ negative predictive value

\begin{tabular}{llllll}
\hline & $0.01 \mathrm{cc}$ & $0.03 \mathrm{cc}$ & $0.06 \mathrm{cc}$ & $0.09 \mathrm{cc}$ & $0.12 \mathrm{cc}$ \\
\hline Sensitivity (\%) & 100.0 & 93.8 & 87.5 & 75.0 & 56.3 \\
Specificity (\%) & 55.6 & 75.6 & 86.7 & 91.1 & 93.3 \\
PPV (\%) & 44.4 & 57.7 & 70.0 & 75.0 & 75.0 \\
NPV (\%) & 100.0 & 97.1 & 95.1 & 91.1 & 85.7 \\
\hline
\end{tabular}

A stenosis of $40 \%$ or greater was selected as positive for "significant" stenosis. Thresholds of $0.03 \mathrm{cc}$ and $0.06 \mathrm{cc}$ have the highest combination of sensitivity and specificity. Statistically, the square roots of these five thresholds provide the same sensitivity, specificity, PPV and NPV so a duplicate table was not included for purposes of brevity

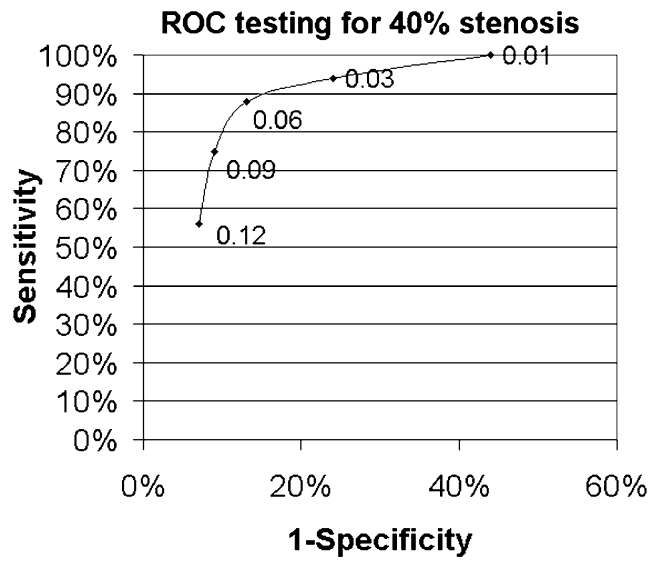

Fig. 2 The ROC curve is composed of five thresholds: 0.01, 0.03, $0.06,0.09$, and $0.12 \mathrm{cc}$. The ROC curve illustrates that the calcium volume thresholds of 0.03 and $0.06 \mathrm{cc}$ are relatively sensitive and specific for detecting a $>40 \%$ ICA stenosis

Using the data from all 117 carotid bifurcations, we computed the correlation coefficient $(r)$ between calcium volume and percent stenosis. This correlation was moderate to strong with $r=0.65$ for all carotids together $(r=0.72$ for right side only, 0.62 for left only, $P<0.0001$ in all cases). The scatter plot in Fig. 3 demonstrates the relationship between percent stenosis and the corresponding measured calcium volume. It was noted that the on the scatter plot the calcium volume appeared to increase faster than the degree of stenosis, and hence calculations were performed for the correlation coefficient $r$ for various permutations of the calcium volumes (square root, cube root, square, etc. of the calcium volume) to find the manipulation of the calcium volume that would produce the greatest correlation with the percent stenosis. We found that the square root of the measured calcium volume had a more linear relationship and hence a stronger correlation to percent stenosis $(r=0.77$ for all carotids together, $r=0.79$ for the right carotids, $r=0.75$ for the left carotids, $P<0.0001$ in all cases) than the actual calcium volume itself. The scatter plot with linear regression curve in Fig. 4 shows the relationship between the percent stenosis and this square root of the calcium volume. We note that the use of the square root of the calcium volume as a threshold to detect for stenosis does not change the sensitivity or specificity of our test when using the square root of the calcium volume and thresholds of $0.01-0.12 \mathrm{cc}$. We also note that in the two scatter plots (Fig. 3, 4), there were many carotids (59 total) where there was no measurable calcium or percent stenosis (calcium volume and percent stenosis both $=0$ ). Thus for clarity, we expanded out the zero point on both axes so that all data points could be visualized. 
Fig. 3 Scatter plot demonstrates correlation between percent ICA stenosis and calcium volume (in cc) of the carotid bifurcation

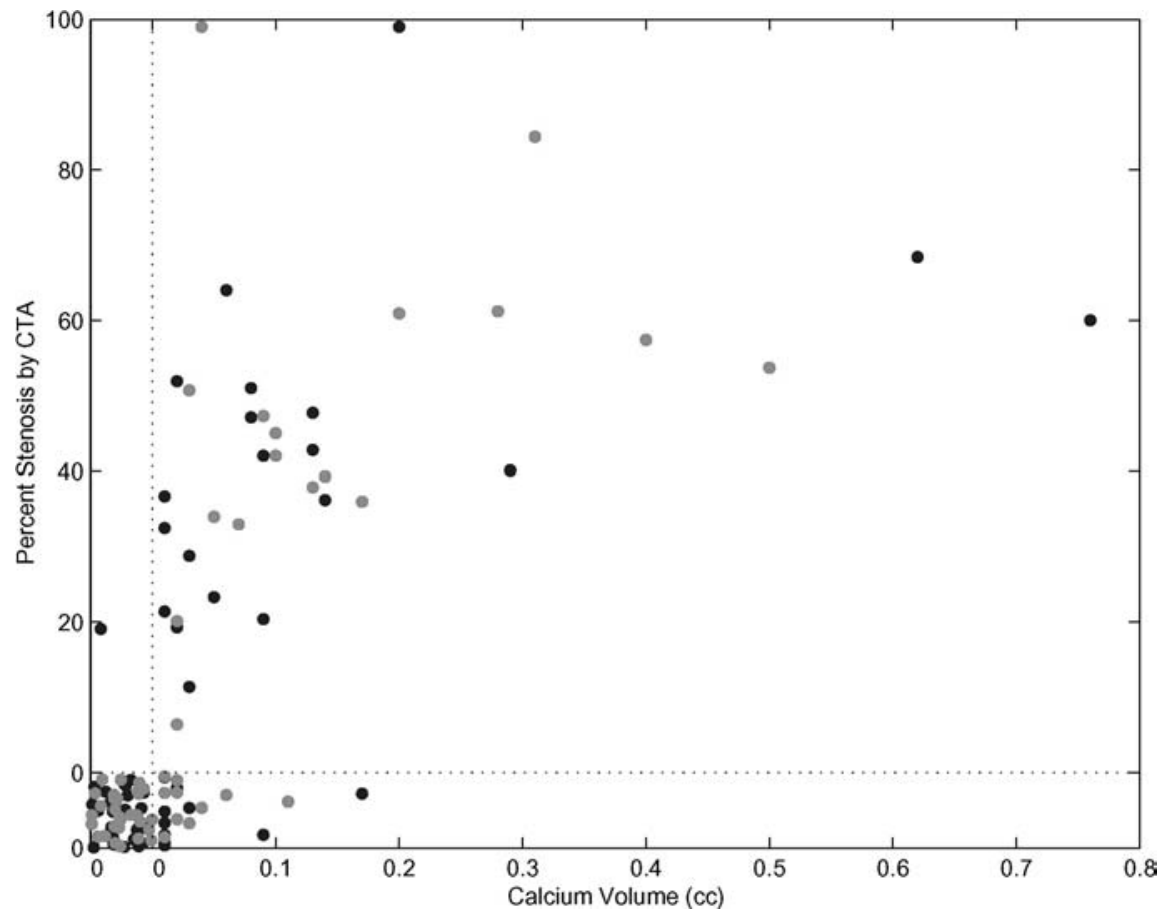

\section{Discussion}

Our paper was designed to test the sensitivity/specificity and feasibility of carotid bifurcation calcium volume measurements in detecting "significant" ICA stenosis, and to correlate those volume measurements with the degree of stenosis. Regarding the use of calcium measurements, the diagnostic use of CT for calcified plaque measurements was initially developed for the detection and screening of patients with clinical coronary artery disease, and significant differences in calcium "score"
Fig. 4 Scatter plot with linear regression curve demonstrates the strong correlation $(r=$ 0.77 ) between percent ICA stenosis and square root of the calcium volume for all 117 carotid bifurcations. Note that 59 carotids were randomized at the zero point (where carotid bifurcation volume $=0$ and percent stenosis $=0$ ) to better illustrate this number of patients and to prevent these values from visually appearing as one point at the $x=0, y=0$ axis

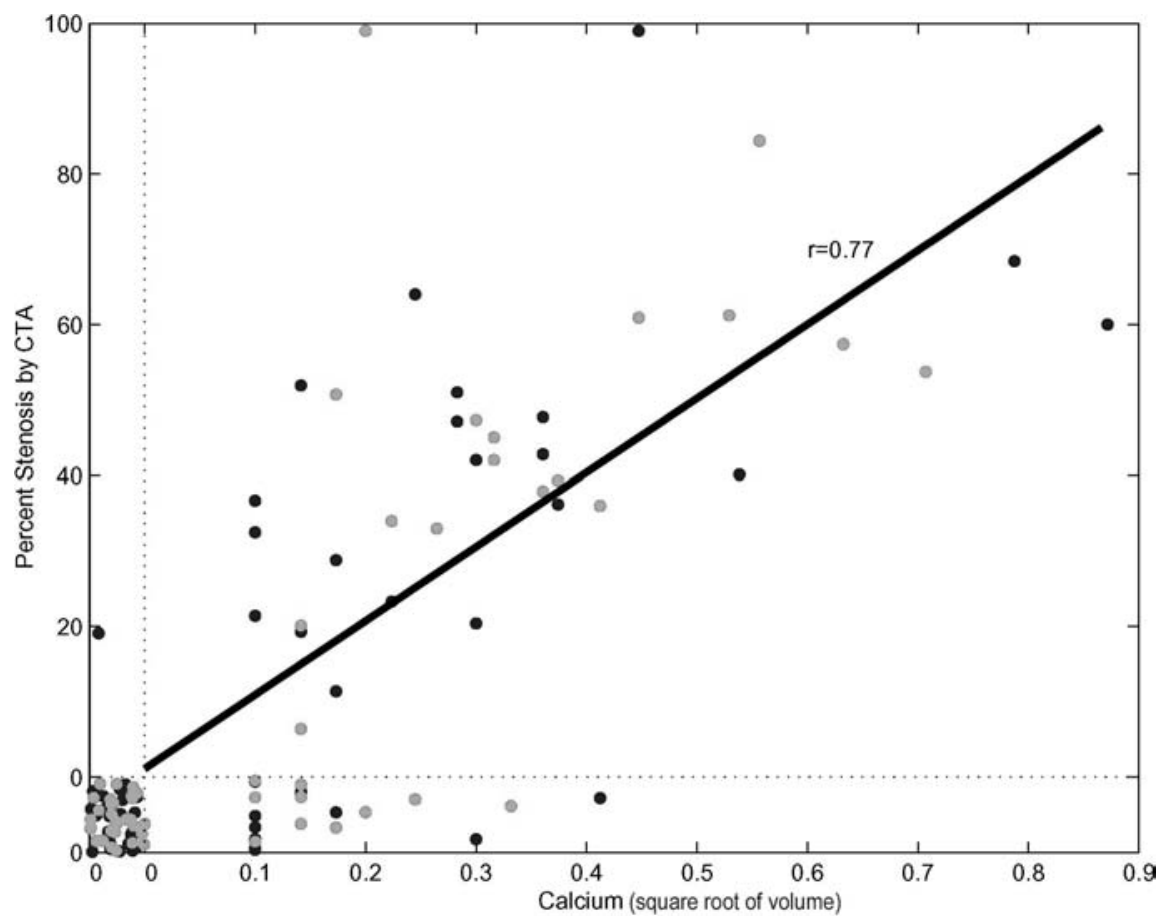


(based on the number, areas and peak CT Houndsfield units of the calcific lesions) were noted between those with versus those without clinical coronary artery disease [15]. Ultrafast CT had already been shown to be more sensitive and specific than fluoroscopy for detecting coronary calcium [16]. The sensitivity and specificity of helical CT in detecting significant/obstructive coronary artery disease (defined as greater than $50 \%$ stenosis) when considering plaque density measurements has been reported previously as 88 and $52 \%$, respectively [5]. Hence, we set out to evaluate if measurements of the amount of bifurcation calcium (in this case measured as a volume) would correlate with ICA stenoses, although we could not use an identical system to that in the coronaries due to the inherent criteria (e.g. multiple vessel evaluation factors into coronary scoring).

In our study of carotid calcium volumes, we obtained sensitivities of 94 and $88 \%$ at 0.03 and $0.06 \mathrm{cc}$, respectively, and specificities of 76 and $87 \%$ for detecting $>40 \%$ stenoses as determined by CTA. On the basis of this preliminary study, use of a calcium volume as a threshold to evaluate for stenosis appears as sensitive and more specific than helical CT and EBCT coronary scoring in detection of "significant" internal carotid luminal stenosis near the bifurcation. In the literature, there has also been a strong correlation between coronary calcium scoring positives on EBCT (using a Houndsfield unit threshold) and degree of coronary stenosis in the involved vessel on autopsy (Pearson's correlation coefficient $r=0.66$ ) [8]. Our study found a relatively strong correlation $(r=0.65)$ between the calcium volume and percent stenosis on CTA. Since we noted that this relatively strong correlation was present, we sought to define a manipulation or equation of the calcium volume that would better correlate with the percent stenosis on CTA (although this would not change the sensitivity and specificity of our test). Our statistician found that the use of the square root of the calcium volume has an even stronger and more linear correlation $(r=0.77)$ with percent stenosis as measured by CTA.

We chose CTA using NASCET-like criteria as a surrogate measure of stenosis over the usual "gold" standard of conventional angiography. This choice permitted our being able to simultaneously acquire CT calcium volume and CTA stenosis measurements in the same patient (who was being scanned for other purposes) in a single evaluation. The $1-\mathrm{mm}$ collimation capability of the multidetector CT scanner allowed this data to be retrospectively reconstructed from the diagnostic neck CT data. Thus, a potential limitation is that we implemented NASCET-like criteria (originally developed for angiography) by using CTA derived measurements as the standard for our study. However, CTA measures of all degrees of stenosis have been previously shown to correlate very strongly ( $r$ correlation coefficient ranging from 0.892 to 0.987 ) with conventional catheter cervicocerebral angiography $[11,13,17]$ and to have a stronger correlation than ultrasound (0.808) compared to conventional catheter cervicocerebral angiography [17]. CTA has been reported as very sensitive $(89 \%)$, specific $(91 \%)$ and accurate $(90 \%)$ in detecting if luminal stenosis is greater than $50 \%$ (versus DSA), but it is less sensitive in characterizing whether the stenosis is within the $50-69 \%$ range $(65 \%)$ or the 70 $99 \%$ range $(73 \%)$ [15]. CTA has also been shown to be accurate (relative to DSA) in characterizing the degree of stenosis even in a "moderate" stenosis (defined as 30 $50 \%)[18]$.

Our study was designed to evaluate if there is a correlation between percent stenosis on CTA and calcium burden, as measured on CT. We obtained our test population from persons who were undergoing neck CT for various clinical reasons, most of which were for evaluating head and neck neoplasms. These patients often have an inherently higher risk and prevalence of atherosclerotic disease compared to the general population, so our test population is likely not truly representative of the general population. Therefore, this could lead to a somewhat elevated rate of detection as well as an element of expectation bias within our studied sample. However, these are patients at high risk for carotid stenosis, and early detection is the intent of this study, Hence, the goal of this study was to evaluate the utility of this examination in those patients at a somewhat higher risk of atherosclertotic carotid disease. We arbitrarily selected 50 years as the threshold age for our "simulated" screening population. However, one could argue that earlier ages in diabetics, hypertensive patients, or patients with hypercholesterolemia could have been included. Also, there may be gender differences in age of onset of carotid disease or differences in age of onset between ethnic groups, which was not accounted for by our study.

We selected $40 \%$ or greater luminal stenosis to be "significant", since ultrasound screening thresholds vary widely and some studies use 40\% [19-21] as the lower threshold to evaluate for stenosis while others use $50 \%$ $[22,23]$ to evaluate for a significant stenosis. Since a hemodynamically significant (i.e. flow-limiting) stenosis has traditionally been considered to be $50 \%$ or greater, we decided on a threshold of $40 \%$ for the purposes of potentially identify the patients at risk before their lesions become flow-limiting, since one of the primary purposes of our study was directed towards the possibility of using the calcium volume measurement as a tool for early detection of carotid disease.

A single observer performed the initial calculations of both stenosis and calcium burden. Hence, interoperator variability was not evaluated for with regard to stenosis measurement, although the calcium volume measurement was relatively automated once the volume was 
sculpted, since the window and level were manipulated within a relatively narrow range of Hounsfield units. We did not notice a significant difference in the calcium volume measured when manipulating the window and level within that range. However, this is a potential source of variability between observers.

We obtained the carotid calcium volumes from studies in which intravenous contrast medium was utilized, thus obligating the observer to segment the calcium from intraluminal contrast via windowing/level manipulation. Calcified plaque was easy to differentiate from intravascular contrast enhancement, but occasionally differentiating noncalcified plaque from vascular enhancement was slightly more time consuming. Therefore, care was taken to accurately measure the stenosis in cases of noncalcified plaque (appearance of noncalcified combined with calcified plaque is demonstrated in Fig. 1f). Since the CTAs of all of the patients in our study were reconstructed from routine neck CTs with intravenous contrast, noncontrast volume measurements were not obtained.

In theory, greater sensitivity for detection of carotid calcium would be attained with noncontrast CT carotid calcium volume measurements, since a single threshold of calcium plaque density could be implemented (in a manner similar to the Hounsfield density threshold in coronary scoring), which could be performed even by a technician. This volume analysis measurement could also potentially become automated by the development of computer software since calcified plaque would stand out more readily from non-enhancing ICA vasculature (and it would not be necessary to visually sculpt a volume as in our study), which was not evaluated here. A non-contrast measurement of carotid calcium volume by CT would also be much faster to review (compared to the volume measurements obtained from our contrast enhanced studies), as much of our time (2-5 min per side) was spent on manipulating the proper plane in which to measure the luminal diameter for stenosis measurement and on segmenting vascular enhancement from calcified plaque. This non-contrast measurement of calcium volumes could serve in a similar fashion to coronary calcium scoring in detecting ICA stenosis and could even be an adjunct to coronary scoring, since patients with atherosclerotic coronary artery disease have been shown to have more rapid progression of extracranial carotid artery disease [24].

Another possibility would be the use of the noncontrast measurement of calcium volumes as an adjunct with carotid ultrasound as a screening tool. This could be derived from the observation that, as with carotid ultrasound (generally in the range of $90-95 \%$ negative predictive value $[25,26])$, the measurements of calcium volumes presented here show relatively high negative predictive values $(97 \%$ at $0.03 \mathrm{cc}$ and $95 \%$ at $0.06 \mathrm{cc})$, which is considered one of the critical criteria of a potential screening method.

Another potential limitation of our study is that common carotid artery (CCA), external carotid artery (ECA), and internal carotid artery calcium were included together in the total volume of calcium measured. Therefore, this volume is not derived solely from the calcified plaque of the ICA, but is actually representative of the overall carotid bifurcation calcified plaque burden. Hence, theoretically a patient could have a high volume of calcium in the CCA (or even ECA) without significant calcified plaque burden or stenosis of the ICA. This distribution of calcification is uncommon (and was not present in our study), since CCA stenosis or occlusion usually involves the ICA as well, but this uncommon variant is a well-described phenomenon in the literature $[27,28]$. Theoretically, in a patient with this pathologic variant, a carotid bifurcation calcium volume obtained by our method may detect a "significant" stenosis, but would actually represent a stenosis of the CCA rather than the intended ICA.

In conclusion, on this preliminary correlation study, measurement of the carotid bifurcation calcium volume (or square root of the calcium volume) demonstrates a relatively strong correlation with percent stenosis on CT angiography in a test population over 50 years of age. In this study, using carotid bifurcation calcium volume thresholds (measured via multidetector CT) appeared to be relatively sensitive and specific and have a high negative predictive value for detection of a greater than $40 \%$ stenosis of the ICA below the skull base.

Future studies may be warranted that would correlate the noncontrast carotid bifurcation calcium volume and the degree of stenosis on ultrasound, CTA, MRA, or conventional catheter angiography. Theoretically, a "score" derived from the noncontrast carotid bifurcation exam could be obtained (whether the calcium volume itself or a numerical manipulation of such) which would be able to identify patients with atherosclerotic disease at high risk for developing flow limiting lesions of the ICA. Hence, the potential would exist for future use of these measurements as a possible non-invasive screening tool. 


\section{References}

1. Robinson JG, Leon AS (1994) The prevention of cardiovascular disease. Emphasis on secondary prevention. Med Clin N Am 78:69-98

2. Alan S, Ulgen MS, Ozturk O, Alan B, Ozdemir L, Toprak N (2003) Relation between coronary artery disease, risk factors and intima-media thickness of carotid artery, arterial distensibility, and stiffness index. Angiology 54:261267

3. Simon A, Giral P, Levenson J (1995) Extracoronary atherosclerotic plaque at multiple sites and total coronary calcification deposit in asymptomatic men. Association with coronary risk profile. Circulation 92:1414-1421

4. Crouse JR, Toole JF, McKinney WM, Dignan MB, Howard G, Kahl FR, McMahan MR, Harpold GH (1987) Risk factors for extracranial carotid artery atherosclerosis. Stroke 18:990996

5. Broderick LS, Shemesh J, Wilensky RL et al. (1996) Measurement of coronary artery calcium with dual-slice helical CT compared with coronary angiography: evaluation of CT scoring methods, interobserver variations, and reproducibility. Am J Roentgenol 167:439-444

6. Woodcock RJ Jr, Goldstein JH, Kallmes DF, Cloft HJ, Phillips CD (1999) Angiographic correlation of CT calcification in the carotid siphon. Am J Neuroradiol 20:495-499

7. Rumberger JA, Sheedy PF, Breen JF, Schwartz RS (1995) Coronary calcium, as determined by electron beam computed tomography, and coronary disease on arteriogram. Circulation 91:1363-1367

8. Simons DB, Schwartz RS, Edwards WD, Sheedy PF, Breen JF, Rumberger JA (1992) Noninvasive definition of anatomic coronary artery disease by ultrafast computed tomographic scanning: a quantitative pathologic comparison study. J Am Coll Cardiol 20:1118-1126

9. Nederkoorn PJ, van der Graaf Y, Hunink MG (2003) Duplex ultrasound and magnetic resonance angiography compared with digital subtraction angiography in carotid artery stenosis: a systematic review (Epub 2003 Apr 10). Stroke 34(5):1324-1332
10. Johnston DC, Goldstein LB (2001) Clinical carotid endarterectomy decision making: noninvasive vascular imaging versus angiography. Neurology 56:1009-1015

11. Cumming MJ, Morrow IM (1995) Carotid artery stenosis: a prospective comparison of CT angiography and conventional angiography. Am J Roentgenol 165:233-235

12. Cinat M, Lane CT, Pham H, Lee A, Wilson SE, Gordon I (1998) Helical CT angiography in the preoperative evaluation of carotid artery stenosis. J Vasc Surg 28:290-300

13. Sameshima T, Futami S, Morita Y, Yokogami K, Miyahara S, Sameshima Y, Goya T, Wakisaka S (1999) Clinical usefulness of and problems with threedimensional CT angiography for the evaluation of arteriosclerotic stenosis of the carotid artery: comparison with conventional angiography, MRA, and ultrasound sonography. Surg Neurol 51:301-309

14. Beneficial effect of carotid endarterectomy in symptomatic patients with high grade carotid stenosis (1991) North American Symptomatic Carotid Endarterectomy Trial collaborators. N Engl J Med 325:445-53

15. Janowitz WR, Agatston AS, Viamonte M Jr (1991) Comparison of serial quantitative evaluation of calcified coronary artery plaque by ultrafast computed tomography in persons with and without obstructive coronary artery disease. Am J Cardiol 68:1-6

16. Agatston AS, Janowitz WR, Hildner FJ, Zusmer NR, Viamonte M Jr, Detrano R (1990) Quantification of coronary artery calcium using ultrafast computed tomography. J Am Coll Cardiol 15:827-832

17. Anderson GB, Ashforth R, Steinke DE, Ferdinandy R, Findlay JM (2000) CT angiography for the detection and characterization of carotid artery bifurcation disease. Stroke 31:2168 2174

18. Binaghi S, Maeder P, Uske A, Meuwly JY, Devuyst G, Meuli RA (2001) Threedimensional computed tomography angiography and magnetic resonance angiography of carotid bifurcation stenosis. Eur Neurol 46:25-34

19. White JE, Russell WL, Greer MS, Whittle MT (1994) Efficacy of screening MR angiography and Doppler ultrasonography in the evaluation of carotid artery stenosis. Am Surg 60:340-348
20. Muller M, Ciccotti P, Reiche W, Hagen T (2001) Comparison of color-flow Doppler scanning, power Doppler scanning, and frequency shift for assessment of carotid artery stenosis. J Vasc Surg 34:1090-1095

21. Arbeille P, Desombre C, Aesh B, Philippot M, Lapierre F (1995) Quantification and assessment of carotid artery lesions: degree of stenosis and plaque volume. J Clin Ultrasound 23:113-124

22. Zwiebel WJ, Austin CW, Sackett JF, Strother CM (1983) Correlation of high-resolution, B-mode and continuous-wave Doppler sonography with arteriography in the diagnosis of carotid stenosis. Radiology 149:523-532

23. Blackshear WM Jr, Lamb SL, Kollipara VS, Anderson JD, Murtagh FR, Shah CP, Farber MS (1984) Correlation of hemodynamically significant internal carotid stenosis with pulsed Doppler frequency analysis. Ann Surg 199:475481

24. Crouse JR 3rd, Tang R, Espeland MA, Terry JG, Morgan T, Mercuri M (2002) Associations of extracranial carotid atherosclerosis progression with coronary status and risk factors in patients with and without coronary artery disease. Circulation 106:2061-2066

25. Fischer GG, Anderson DC, Farber R, Lebow S (1985) Prediction of carotid disease by ultrasound and digital subtraction angiography. Arch Neurol 42:224-227

26. Mittl RL Jr, Broderick M, Carpenter JP et al. (1994) Blinded-reader comparison of magnetic resonance angiography and duplex ultrasonography for carotid artery bifurcation stenosis. Stroke 25:4-10

27. Keller HM, Valavanis A, Imhof HG, Turina M (1984) Patency of external and internal carotid artery in the presence of an occluded common carotid artery: noninvasive evaluation with combined cerebrovascular Doppler examination and sequential computer tomography. Stroke 15:149-157

28. Riles TS, Imparato AM, Posner MP, Eikelboom BC (1984) Common carotid occlusion. Assessment of the distal vessels. Ann Surg 199:363-366 